\title{
A pain in the eye: what's the category?
}

R ecording electrical signals from the eye of a living mouse may become a little easier with the use of an electrode mesh that can be injected into an animal's eye. The basic technique, as recently described ${ }^{1}$, is to perform an intravitreal injection into the rear of the eye where the mesh then unfolds onto the retina. Wires attached to the mesh and an external electrical recording device exit the eye at the lateral canthus.

The new technique was being proposed for use at Great Eastern University where Dr. Tom Villanueva, the attending veterinarian, was presenting the details of the technique to the IACUC. Dr. Brad Collins, an IACUC member, interrupted him to ask if the wires that exit from the eye are painful to the mouse. "I've seen videos of the surgery and aftercare" said Villanueva, "and it doesn't appear to bother them. They don't paw at the eye, they seem to act normally in the cage, they have normal ocular pressure and a normal pupillary reflex."
"That may be true," said Collins, "but if I had a wire protruding from the corner of my eye, I wouldn't be a happy camper. I don't know how much it would hurt, but I'll bet it would bother me. What pain category is being proposed for this protocol?"

"USDA category D," responded Villanueva. "The surgery is performed under anesthesia, and we will be giving buprenorphine for three days post-op."

"Well, I'm still concerned," Collins said. "If we believe that a procedure that's painful or distressful to a human is likely to be painful or distressful to a mouse, then I think this should be category E, due to unalleviated distress from the wires attached to its eye and acutely altered vision from the presence of the mesh."

"I disagree," Villanueva answered. "As far as I know this procedure has never been performed on humans, so I don't see how you can make that correlation.
The investigator was trained on the technique at a lab where it's being used and he knows what he's doing, so I think it's best to let the committee discuss the protocol and vote on it."

If you were a member of the Great Eastern IACUC, are there additional questions you would ask? From the information currently available, which pain category do you think is appropriate for this study?

\section{Jerald Silverman}

University of Massachusetts Medical School, Worcester, Massachusetts, USA. e-mail: Jerald.Silverman@umassmed.edu

Published online: 20 March 2019

https://doi.org/10.1038/s41684-019-0262-5

References

1. Hong, G., Fu, T.-M., Qian, M., Viveros, R. D., Yang, X., Zhou, T., Lee, J. M., Park, H.-G., Sanes, J. R. \& Lieber, C. M. Science 360, 1447-1451 (2018).

\section{Evaluation is more important than categorization}

T his scenario exemplifies a common debate: to what pain category does a study belong? For this study, satisfactory arguments can be made for classifying animals into either category $\mathrm{D}$ or $\mathrm{E}$; the authors' knee jerk reaction is to label it E for the same reasons voiced by Dr. Collins. But we suggest instead that we should argue the utility of classifying pain in nonUSDA covered species. Unless assignment to a pain category dictates specific care, monitoring, or interventions, categorization is not directly relevant to the charge of the IACUC: to ensure the wellbeing of animals used in research. The IACUC should focus on helping the investigator develop plans to learn how the device will affect the animal, and for managing the animals through thorough protocol review, veterinary involvement, and post-approval monitoring ${ }^{1}$.

This should start with information gathering during protocol review. Has the PI (or his or her staff) performed this procedure before, and are their clinical outcomes consistent with the video evidence recounted by Dr. Villanueva? Have adverse consequences occurred, including early removal from study? If personnel are not experienced, can clinical or histologic outcomes of practice animals be used to develop monitoring and intervention criteria? A veterinarian should be involved in the review of these responses with the IACUC, as well as care of the practice animals to help evaluate animal outcomes. $\mathrm{He}$ or she can then propose criteria for interventions, and train lab personnel in recognizing those criteria. Finally, the IACUC can employ post-approval monitoring to evaluate outcomes. Do animals exhibit signs of good welfare, and is there evidence of veterinary involvement and support? Have there been adverse outcomes? These questions illustrate the process that we propose is the crux of the issue: how to provide for the welfare of animals undergoing a procedure that may or may not cause chronic pain or distress. We believe the answer lies in a premeditated but fluid approach to evaluate and intervene based on outcomes, aims, and communication.

But at the Great Eastern University, a pain category must be named. If one relies on USDA examples by procedure, we propose the mesh insertion would fall into $\mathrm{D}$, as it subjectively feels similar to survival surgery, orbital blood collection, and needle biopsy $^{2}$. The presence of the exiting wire could approximate the category E example of ocular or skin irritancy testing if we assume the exiting wire is irritating, though we do not know this would be painful or distressing ${ }^{2}$. The case for category E could be strengthened depending on the animals' cumulative experience. Even if the mesh insertion is the most severe procedure proposed, it alone should not dictate the pain category since factors like single housing, repeated anesthesia or restraint, or a specific phenotype can contribute to this classification.

Regardless of what categorization the IACUC agrees upon, we maintain that the more critical aspect of this scenario is the actions taken by the IACUC, the veterinary team, and the research staff to protect the welfare of the animals used in the study.

\section{Felicia D. Duke Boynton* and}

AlexaEdmunson

University of Minnesota, Minneapolis, MN, USA.

*e-mail:fduke@umn.edu

Published online: 20 March 2019

https://doi.org/10.1038/s41684-019-0263-4

References

1. Animal Welfare Regulations 2012.9 CFR $\$ 2.31$

2. USDA Pain Levels. https://www.esf.edu/animalcare/documents/ USDApainLevels.pdf 